Especial

\title{
Aplicación del proceso de atención de enfermería a la salud laboral
}

\section{Application of the process of care for occupational health nursing}

\author{
Jesús González Sánchez \\ Servicio de Prevención de Riesgos Laborales. MC - Prevención. Salamanca. España. \\ Recibido: 09-02-11 \\ Aceptado: 22-02-11 \\ Correspondencia \\ Jesús González Sánchez \\ MC - Prevención Salamanca \\ Plaza Mariseca, s/n (esquina Calle del Hornazo) \\ 37003 - Salamanca. España \\ Tfno: 923100137 \\ e-mail: igonzalezsa@mc-prevencion.com
}

\section{Resumen}

La salud laboral, es uno de los campos en los que tiene su actuación el Proceso de Atención de Enfermería, pues nos hallamos en un ámbito profesional de prestación de cuidados y atención a los pacientes, en este caso a los trabajadores. Su aplicación va a suponer la utilización del método científico por parte del Enfermero del Trabajo a su praxis diaria, abarcando para ello cinco áreas de actuación: preventiva, asistencial, docente e investigadora, administrativa y gestora. El objetivo fundamental de la aplicación de esta herramienta enfermera de trabajo, será el de constituir una estructura que pueda cubrir, individualizándolas, las necesidades del trabajador que acude a un servicio de prevención, mejorando la eficiencia y la eficacia del trabajo y favoreciendo una curación más fácil y rápida. Esta aplicación es ciertamente novedosa, pues tratamos un esquema tradicional y consensuado de Enfermería, sobre una nueva especialidad en la salud laboral.

Med Segur Trab (Internet) 2011; 57 (222) 15-22

Palabras clave: proceso de atención enfermera, salud laboral, enfermería del trabajo, diagnóstico de enfermería, plan de cuidados, prevención.

\begin{abstract}
Occupational health is an area in which Nursing Care Process has an important role, as we are in a professional environment in charge of the provision of patient care, in this case workers. Its implementation will involve the use of a scientific method by the Labor Nurse in her daily practice, involving these five areas: prevention, care, teaching and research, administrative and management. The main objective of the implementation of this tool will be to provide a structure that can cover individualized needs of workers who demand the attention of the prevention area, improving efficiency and effectiveness of work and an easier and higher rate of the healing process. This application is certainly new, as we apply a traditional pattern of Nursing in a new area of occupational health.
\end{abstract}

Med Segur Trab (Internet) 2011; 57 (222) 15-22

Keywords: nursing care process, occupational bealth, occupational nursing, nursing diagnosis, care plan, prevention. 


\section{INTRODUCCIÓN}

Los cambios e innovaciones que se han producido en la especialidad de la Enfermería del Trabajo en los últimos años, han sido claves, ampliándose su campo de actuación, autonomía y responsabilidad de forma considerable.

Según se establece en la Orden SAS/1348/2009, de 6 de mayo ${ }^{1}$, por la que se aprueba y publica el programa formativo de la especialidad de Enfermería del Trabajo, al futuro especialista en Enfermería del Trabajo, se le van a exigir unas competencias específicas abarcando cinco grandes áreas de actuación: Preventiva, Asistencial, Legal y Pericial, Gestora y Docente e Investigadora. Para llevar a cabo estas competencias, vamos a poder ayudarnos de la aplicación del Proceso de Atención de Enfermería, como vamos a exponer, en todas y cada una de sus fases.

También es importante destacar, que según dicha Orden SAS/1348/2009, de 6 de mayo $^{1}$, el futuro especialista en Enfermería del Trabajo, estará capacitado para desarrollar su actividad laboral, al menos, en los siguientes ámbitos:

a) Servicios de prevención de riesgos laborales en sus distintas modalidades.

b) Servicios de prevención/servicios médicos de empresa/unidades relacionadas con un medio laboral concreto (aeroespacial, subacuático, marítimo, deportivo, de inspección, educativo....).

c) Centros sanitarios, servicios, unidades, instituciones de las administraciones públicas y cualquier otra entidad pública o privada con competencias en prevención de riesgos laborales/salud laboral.

d) Gabinetes de salud laboral en las administraciones públicas.

e) Centros de docencia e investigación en salud laboral y enfermería del Trabajo.

f) Servicios de salud medioambiental.

De forma general, la prestación de servicios de los especialistas en Enfermería del Trabajo, viene a desarrollarse en empresas y servicios de prevención tanto ajenos como propios, generalmente en el sector privado, ya que la existencia de sistemas de selección y oposiciones de esta especialidad es bastante escasa a día de hoy. Con la reciente aprobación de la especialidad y el comienzo de la formación de especialistas a partir de este año, contribuirá a que estos procesos de selección se incrementen a medida que las necesidades y la presencia de especialistas formados crezcan. En la página web del Ministerio de Sanidad y Política Social ${ }^{2}$, se expone una clasificación de la actividad sanitaria de los servicios de prevención, en función de los riesgos laborales en el marco de las actividades de prevención (Figura 1), en la cual se refleja la importancia de la participación de Enfermería del Trabajo junto a otros profesionales no sanitarios, como son los técnicos en prevención de riesgos laborales.

Figura 1. Clasificación de la actividad sanitaria en la salud laboral.

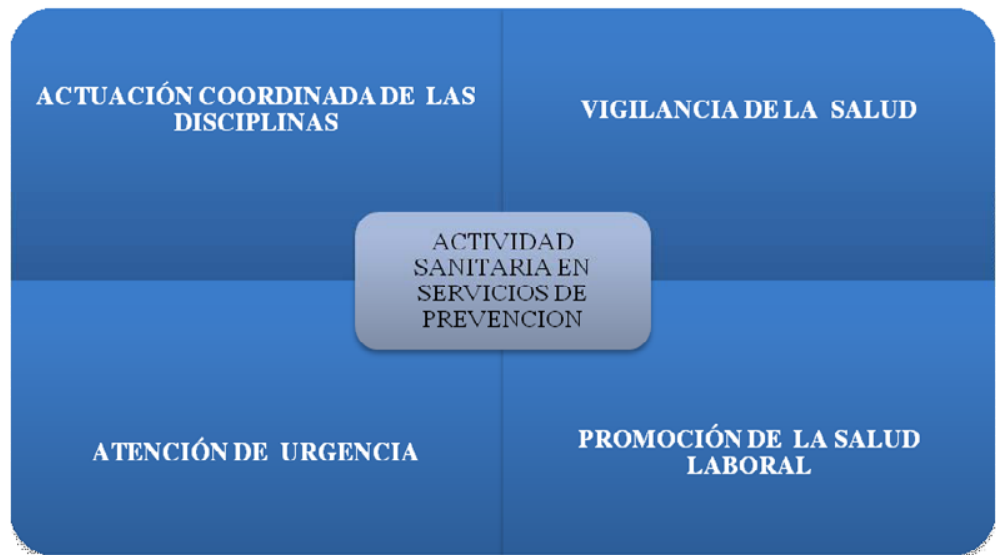


La realización de la mayoría de estas actividades, se van a desarrollar de forma habitual por el Enfermero del Trabajo en las distintas empresas y servicios de prevención, como por ejemplo:

1. Participación en los exámenes de salud laboral.

2. Realización de actividades docentes e investigadoras.

3. Ejecución de campañas de vacunación.

4. Prestación de cuidados de urgencia y primeros auxilios.

5. Colaboración junto con el técnico en prevención, en la identificación y evaluación de riesgos de los trabajadores.

La prevención de los riesgos, así como la mejora de las condiciones de trabajo, debe de ser un objetivo permanente y prioritario de la dirección de la empresa ${ }^{3}$.

La figura actual del especialista en Enfermería del Trabajo es crucial, para poder llevar a cabo una adecuada vigilancia de la salud de los trabajadores, se trata de un profesional que debe estar altamente cualificado y con suficientes conocimientos para desarrollar su cometido, así como percibir e identificar alteraciones y riesgos que el trabajador pueda haber adquirido como consecuencia del desarrollo de su puesto de trabajo.

Con el objetivo de prestar una atención de calidad al trabajador, la Enfermería del Trabajo dispone de una herramienta útil para ello, el Proceso de Atención de Enfermería (PAE) aplicado a la salud laboral, teniendo en cuenta que todas las personas tienen necesidades de cuidados, que el estado de salud es un condicionante para los mismos y que cada ser humano responde de manera particular a esas necesidades ${ }^{4}$.

La participación de Enfermería del Trabajo en el diagnóstico precoz de patologías de origen laboral tiene una doble vertiente: por un lado, la aplicación del PAE en las áreas asistencial, de gestión, legal y pericial es una herramienta útil para la colaboración en ese diagnóstico precoz (sobre todo con las fases iniciales de Valoración y Diagnósticos Enfermeros) y por otro lado, en las áreas de prevención, docencia e investigación, utilizando los datos aportados de pruebas complementarias y resultados de los test y cuestionarios que complementan los protocolos específicos de los exámenes de salud laboral. Vamos a exponer como esta herramienta enfermera, tradicional y estructurada, tiene aplicación en todas y cada una de sus fases, así como las múltiples ventajas que podemos lograr de su utilización en el ámbito de la salud laboral.

Pero la responsabilidad y funciones del Enfermero del Trabajo, no deben limitarse a la vigilancia de la salud y a proporcionar la atención sanitaria de urgencia en el lugar de trabajo, sino que también debe extender su actividad a las circunstancias que afecten a la salud de los trabajadores, a formar e informar a los mismos, a promocionar la salud en el lugar de trabajo colaborando asimismo con el Sistema Nacional de Salud y con las Autoridades Sanitarias.

\section{OBJETIVOS}

Como objetivo general planteamos la demostración de la aplicación en el ámbito laboral y dentro de las competencias y funciones de la Enfermería del Trabajo, de las cinco etapas que componen el Proceso de Atención de Enfermería, como herramienta enfermera y esquema estructurado de prestación de cuidados, en este caso al trabajador.

Entre los objetivos específicos se encuentran:

1. Analizar la prestación de cuidados y atención dada al trabajador.

2. Valorar aspectos y situaciones que se benefician o potencian con la aplicación de este proceso de atención, como la comunicación con el trabajador así como su colaboración en alguna toma de decisión y aspectos relacionados con su salud. 
3. Intentar relacionar con su aplicación, una mejora de atención y mayor calidad de cuidados prestada al trabajador.

4. Detectar y analizar mejoras en aspectos relacionados con el registro y documentación en el servicio de prevención, de actos realizados por el especialista en Enfermería del Trabajo.

5. Facilitar el análisis y estudio de datos e información sanitaria, que favorezca la realización de estudios epidemiológicos y trabajos de investigación.

\section{DESARROLLO DEL PROCESO DE ATENCIÓN DE ENFERMERÍA (PAE), EN SALUD LABORAL}

Atendiendo a la definición del PAE: Aplicación del método científico en la práctica asistencial que nos permite a los profesionales de Enfermería prestar cuidados que se nos demandan, de una forma estructurada, homogénea, lógica y sistemática ${ }^{20}$, podemos afirmar que en nuestro caso, se trataría de la prestación de cuidados en los que irán incluidas actividades asistenciales, docentes, gestoras, investigadoras y preventivas por parte del Enfermero del Trabajo, hacia los trabajadores de su empresa o servicio de prevención, permitiéndonos realizar nuestra tarea de forma más completa y eficaz.

La doctrina tradicional viene afirmando que, el Proceso de Atención de Enfermería (PAE) se compone de cinco grandes etapas: Valoración, Diagnóstico, Planificación, Ejecución y Evaluación, (Figura 2).

Figura 2. Etapas del Proceso de Atención de Enfermería

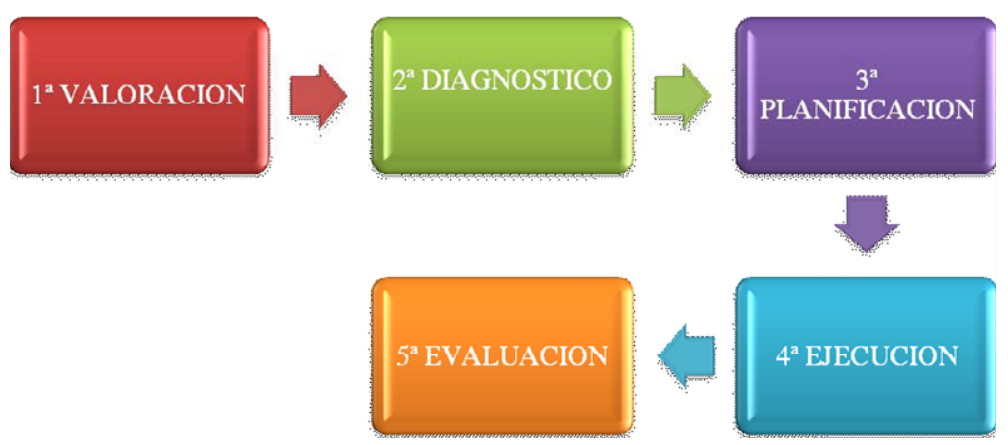

Nuestra pretensión es extrapolar el PAE al ámbito de la salud laboral en la práctica enfermera, desde el convencimiento en que su aplicación, garantiza la seguridad y calidad de las actuaciones de Enfermería del Trabajo.

Es importante destacar que, el Enfermero del Trabajo, durante todo el desarrollo del Proceso de Atención de Enfermería, debe de abarcar los siguientes apartados:

1) Identificar las necesidades reales y potenciales del trabajador.

2) Establecer el plan de cuidados individual necesario.

3) Actuar para cubrir y resolver los problemas, prevenir o curar la enfermedad.

\section{ENFERMERÍA DEL TRABAJO EN LA VALORACIÓN DEL PAE}

En esta etapa vamos a recoger y examinar la información sobre el estado de salud del trabajador, buscando evidencias de funcionamiento anormal o factores de riesgo que pueden generar problemas de salud. Normalmente a esta fase se la califica como la de mayor importancia, para lo cual debemos de poner en marcha toda nuestra capacidad, experiencia y conocimientos. 
El término "vigilancia de la salud de los trabajadores" engloba una serie de actividades, referidas tanto a individuos como a colectividades y orientadas a la prevención de los riesgos laborales, cuyos objetivos generales tienen que ver con la identificación de problemas de salud y la evaluación de intervenciones preventivas ${ }^{5}$.

En esta primera etapa de valoración, utilizaremos una serie de herramientas, que son ${ }^{6}$ :

- Entrevista

- Observación

- Exploración, mediante la inspección, auscultación, palpación y percusión.

Respecto a la entrevista con el trabajador, la efectuaremos en la anamnesis y cuestionarios existentes en cada examen de la salud, que dependerá del puesto de trabajo desarrollado y del protocolo aplicado, teniendo en cuenta los riesgos laborales a los que el trabajador está expuesto.

En la Observación, se debe de prestar atención a la lesión que el trabajador tenga: ya sea un corte, herida, contusión, a las características de las mismas: si está inflamada, infectada, si hay presencia de cuerpos extraños o de hematoma, supuración de algún tipo, si precisará sutura, y también observar el estado en que se encuentre el trabajador en el momento de su presencia en el centro asistencial, valorando de forma rápida y concreta, su estado general, atendiendo a datos como su estado de conciencia, coloración e integridad de la piel, presencia o ausencia de dolor, higiene y estado de la zona lesionada.

Y por último, la exploración mediante actos como la toma de tensión arterial, valoración de la frecuencia cardíaca y temperatura.

Todos estos datos los anotaremos en la historia clínico-laboral, que es el documento que recoge toda la información y documentación relativa a la vigilancia y control de la salud de los trabajadores ${ }^{7}$, datos de anamnesis, exploración clínica, control biológico y estudios complementarios en función de los riesgos inherentes al trabajo. También se hará constar una descripción detallada del puesto de trabajo, el tiempo de permanencia en el mismo, los riesgos detectados en el análisis de las condiciones de trabajo, y las medidas de prevención adoptadas para ello ${ }^{8}$.

Debemos de tener en cuenta la existencia de la Ley $41 / 2002^{9}$, básica reguladora de la autonomía del paciente y de derechos y obligaciones en materia de información y documentación clínica, vinculante también para el ámbito de la salud laboral. En esta ley se describen artículos claves del recto proceder sobre contenidos que debe de tener la historia clínico laboral del trabajador, máxime cuando la Enfermería viene siendo responsable de la guarda y custodia de la mayoría de la documentación clínica.

\section{ENFERMERÍA DEL TRABAJO EN EL DIAGNÓSTICO DEL PAE}

El Enfermero del Trabajo es un profesional que puede y debe de utilizar los diagnósticos enfermeros en el campo en el que desarrolla sus funciones y competencias, para una mejor y más eficaz atención enfermera hacia el trabajador, en las múltiples situaciones y problemas con los que se puede encontrar. La utilización de estos diagnósticos enfermeros en el ámbito de la salud laboral, los convierten en una pieza clave para combatir las patologías en este contexto, adquiriendo una importancia especial el uso de los diagnósticos potenciales, ya que al hacerlo, el Enfermero del Trabajo va a poder detectar situaciones problemáticas antes de que se produzcan y por lo tanto se podrán tomar medidas preventivas en el trabajador para evitar su aparición. Por ello, los diagnósticos potenciales tienen una enorme importancia en el campo de la prevención, y su uso contribuirá de forma clave en el beneficio del trabajador.

Los diagnósticos que el Enfermero del Trabajo va a poder enunciar, deben de partir de actividades Independientes que lleve a cabo en un servicio de prevención, como por 
ejemplo: realización de campañas de vacunación, cumplimentación de los apartados de enfermería de la historia clínico-laboral, seguimiento y valoración de heridas o realización de actividades de investigación y/o formativas a los trabajadores. Se trata pues de situaciones que debe de saber afrontar con autonomía y responsabilidad, procurando al trabajador una atención enfermera integral y de calidad.

\section{ENFERMERÍA DEL TRABAJO EN LA PLANIFICACIÓN DEL PAE}

Para alcanzar los objetivos previstos de la vigilancia de la salud, es necesaria una adecuada planificación conforme a los riesgos laborales a los que están expuestos los trabajadores ${ }^{10}$.

En esta fase se trata de establecer y llevar a cabo unos cuidados de enfermería, que conduzcan al trabajador a prevenir, reducir o eliminar los problemas detectados.

Para ello, es necesario establecer primero una serie de objetivos, que nos ayuden a guiar y dar forma a la planificación, como ${ }^{11}$ :

- Favorecer unos cuidados individualizados.

- Garantizar una adecuada comunicación.

- Lograr una continuidad asistencial

- Proporcionar las pautas de evaluación de la asistencia prestada al trabajador.

El Enfermero del Trabajo debe de tener en cuenta que, su planificación debe de estructurarse en tres fases ${ }^{11}$ :

\section{1) Orden de prioridades en el tratamiento de los problemas.}

Respecto a esta fase, se recomienda que un plan de cuidados correcto debe contemplar por un lado los diagnósticos de Enfermería y por otro lado los problemas clínicos existentes. Después habrá que priorizar los cuidados, identificando correctamente cuales son más urgentes y prioritarios para la salud del trabajador.

\section{2) Objetivos a conseguir.}

El personal de Enfermería debe de centrarse en la toma de las medidas preventivas oportunas, orientarse en el estudio y vigilancia del diagnóstico precoz, vigilar la evolución y tomar conjuntamente con el médico del trabajo, las medidas correctoras oportunas.

Para elaborar dichos objetivos, el Enfermero del Trabajo debe de tener en cuenta el patrón funcional o la respuesta humana alterada, y centrar los objetivos directamente en el trabajador.

\section{3) Acciones de Enfermería.}

En esta fase se ponen en marcha las Intervenciones de Enfermería, que son los cuidados que van a realizar, en nuestro caso, dirigidas a promover pautas de comportamiento favorecedoras de la salud, preventivas de la enfermedad y protectoras de los riesgos medioambientales. Dichas actividades conformarán el conjunto denominado "Educación Sanitaria”. El objetivo principal de esta educación es el de estimular al trabajador a adoptar y mantener hábitos laborales inocuos, utilizar el equipo de trabajo adecuadamente y tomar sus propias decisiones para proteger su salud y mejorar las condiciones de trabajo $^{12}$.

\section{ENFERMERÍA DEL TRABAJO EN LA EJECUCIÓN DEL PAE}

En esta etapa se pone en práctica el plan de cuidados, teniendo en cuenta siempre unas prioridades en base a las necesidades y posibilidades del trabajador. 
La puesta en marcha de estas actividades, va a requerir que el profesional de Enfermería realice a su vez una serie de acciones encaminadas a lograr una mayor eficacia en esta fase de ejecución ${ }^{13}$ :

- Continuar con la recogida y valoración de datos.

- Realizar las actividades de enfermería.

- Anotar los cuidados que se prestan.

- Mantener el plan de cuidados actualizado.

Los registros de enfermería constituyen una parte fundamental de la asistencia sanitaria, estando integrados en este caso, en la historia clínica laboral del Trabajador, lo que conlleva unas repercusiones y responsabilidades de índole profesional y legal, que precisa llevarlos a la práctica con el necesario rigor científico, que garantice la calidad de los mismos.

\section{ENFERMERÍA DEL TRABAJO EN LA EVALUACIÓN DEL PAE}

Este proceso de evaluación consta de dos partes:

1) Recogida de datos sobre el estado de salud/problema/diagnóstico que se quiere evaluar. Se trata de valorar y recoger información respecto a la situación actual y final de la lesión o problema que padecía el trabajador.

2) Comparación con los resultados esperados y un juicio sobre la evolución del trabajador hacia la consecución de los resultados esperados, comprobando si los resultados finales coinciden con los esperados o por el contrario no se han logrado los objetivos establecidos. En caso de ser así, el trabajador quedará incapacitado en el grado que por su estado le corresponda, pudiendo ser una incapacidad permanente parcial, total, absoluta o bien adjudicándose al trabajador la situación de gran invalidez, si las secuelas que le queden por su accidente de trabajo o enfermedad profesional así lo requieran.

\section{CONCLUSIONES}

El Proceso de Atención de Enfermería, tiene aplicación en todas y cada una de sus etapas en el ámbito de la salud laboral, favoreciendo:

- El Enfermero del Trabajo, como especialista del ámbito de la salud, viene obligado a realizar sus funciones enfermeras, acorde al grado de diligencia exigible a una mayor cualificación profesional.

- Continuidad de la atención al trabajador, puesto que la característica dinámica de este Proceso de Atención de Enfermería, obliga a trabajar sobre las situaciones nuevas que puedan afectar al trabajador, así como conocer los progresos o recaídas de forma inmediata, evitando sorpresas desagradables tanto para la Enfermero del Trabajo como para el trabajador.

- Participación por parte del trabajador en la toma de decisiones y colaboración para su propia salud y mejora de su lesión, siempre que esto sea posible y no dificulte el proceso de curación.

- Prestación de cuidados con más calidad, aumentado la eficiencia y la eficacia del trabajo, propiciando una curación más fácil y rápida, así como favorecer la comunicación permitiendo que el Enfermero del Trabajo tenga una mejor relación con el trabajador, facilitando así el tratamiento y resultados más positivos y seguros. 


\section{REFERENCIAS BIBLIOGRÁFICAS}

1. Orden SAS/1348/2009, de 6 de mayo, por la que se aprueba y publica el programa formativo de la especialidad de Enfermería del Trabajo, BOE núm. 129, de 28 de Mayo.

2. Ministerio de Sanidad y Política Social. Vigilancia de la Salud de los trabajadores. Disponibleen:http:// www.msc.es/ciudadanos/saludAmbLaboral/saludLaboral/vigiTrabajadores/home.htm

3. Sanz-Gallén, P.;Izquierdo, J.; Prat Marín, A.; Manual de Salud Laboral. Barcelona: Springer-Verlag Ibérica; 1996.

4. Benavent Garcés, $M^{a}$ Amparo; Ferrer Ferrandis, Esperanza; Francisco del Rey, Cristina; Fundamentos de Enfermería. $2^{a}$ Edición. Valencia: DAE; 2009.

5. Ministerio de Sanidad y Política Social. Vigilancia de la Salud de los trabajadores Disponibleen:http:// www.msc.es/ciudadanos/saludAmbLaboral/saludLaboral/vigiTrabajadores/home.htm

6. Vázquez Chozas, José María; Muñoz González, Asunción; El Proceso de Atención de Enfermería, Teoría y Práctica. Alcalá de Guadaíra, Sevilla: MAD;2007.

7. Orden de 07-07-99 de la Consejería de Sanidad, de la historia clínico-laboral. D.O.C.M. n 49 de 23 de julio de 1999.

8. Real decreto. 39/97 de 17 de enero, por el que se aprueba el Reglamento de los Servicios de Prevención. BOE núm. 27 de 31 enero.

9. Ley 41/2002, básica reguladora de la autonomía del paciente y de derechos y obligaciones en materia de información y documentación clínica. BOE núm. 274 de 15 noviembre.

10. Calvo Sánchez, María Dolores; Enfermería del Trabajo, Serie de Cuidados Avanzados. Madrid: DAE; 2008.

11. Serrano Parra, D.; Garrido Abejar, M.; Máster de Enfermería, Enfermería Fundamental. Barcelona: Masson; 2003.

12. Organización Mundial de la Salud. Detección Precoz de Enfermedades Profesionales. Madrid: Gráficas Reunidas; 1987.

13. Alfaro-LeFevre, Rosalinda; Aplicación del Proceso Enfermero, guía paso a paso. $4^{a}$ Edición. Barcelona: Springer - Verlag Ibérica; 2000.

IIIIIIIIIIIIIIIIIIIIIIIIIIIIIIIIIIIIIIIIIIIIIIIIIIIIIIIIIIIIIIIIIIIIIIIIIIIIIIIIIIIIIIIIIIIIIIIIIIIIIIIIIIIIIIIIIIIIIIIIIIIIIIIIIIIIIIIIIIIIIIIIIIIIIIIIIIIIIIIIIIIIIIIIIIIIIIIIIIIIIIII 